\title{
Toxigenic and zoonotic Corynebacterium ulcerans emerges from widespread hedgehog
} disease

Authors: An Martel, Filip Boyen, Jörg Rau, Tobias Eisenberg, Andreas Sing, Anja Berger, Koen Chiers, Sarah Van Praet, Serge Verbanck, Muriel Vervaeke, Frank Pasmans

\section{Affiliations:}

Ghent University, Merelbeke, Belgium (A. Martel, F. Boyen, K. Chiers, S. Van Praet, S. Verbanck, F. Pasmans)

Chemical and Veterinary Analysis Agency Stuttgart, Fellbach, Germany (J. Rau)

Hessian State Laboratory, Giessen, Germany (T. Eisenberg)

Bavarian Health and Food Safety Authority (LGL), Oberschleißheim, Germany (A. Sing, A. Berger)

Agency for Nature and Forests, Belgium (M. Vervaeke)

Address for correspondence: An Martel, Wildlife Health Ghent, Department of Pathology, Bacteriology and Avian Diseases, Faculty of Veterinary Medicine, Ghent University, Salisburylaan 133, 9820 Merelbeke, Belgium. Email: an.martel@ugent.be

\begin{abstract}
Toxin-producing Corynebacterium ulcerans, causing diphtheria in humans, were isolated from 53 diseased hedgehogs during spring 2020 in Belgium. Isolates showed low levels of acquired antimicrobial resistance. Pronounced strain diversity suggests emergence from an endemic situation. These findings stress the need for raising public awareness and improved wildlife disease surveillance.
\end{abstract}

\section{Widespread occurrence of Corynebacterium ulcerans in hedgehogs with ulcerative skin}

\section{disease.}

During May and June 2020, we obtained 60 isolates of C. ulcerans from 81 diseased or dead hedgehogs (Erinaceus europaeus) that were presented with ulcerative skin lesions by the public to four animal rescue centers across northern Belgium (i.e. Flanders) (Figure 1). The bacterium was isolated from ulcers or abscesses on the head or limbs of 53 hedgehogs, all of them being adult males. From six animals, more than one isolate was obtained from different lesions. Although C. ulcerans was most often isolated these lesions yielded abundant, poly- 
bacterial growth (Table 1). Systematic post mortem examinations of nine animals showed good body condition of the animals, with multiple cutaneous ulcers on the head and limbs. Histopathology from the skin of these animals revealed extensive ulcerative dermatitis with phlegmonous inflammation, sometimes extending in the subcutis and even underlying skeletal muscles. Intralesional bacteria as well as fragments of plant material were noticed. Four animals showed an interstitial pneumonia, one animal suffered from absceding hepatitis and in one animal, a fibrinosuppurative epicarditis with intralesional bacteria was noticed. All animals had extramedullar hematopoiesis in the liver and spleen. Despite presence of several other pathogens such as fly maggots (myiasis, $n=5)$, Sarcoptes scabiei $(\mathrm{n}=1)$, Herpesvirus $(\mathrm{n}=2)$, Caparinia spp. $(\mathrm{n}=1)$ and Cresonema striatum $(\mathrm{n}=2)$, no consistent evidence for other, primary disease causes was found. While evidence does not suffice to attribute a causal role for $C$. ulcerans in the lesions observed, its widespread and high-level occurrence in diseased male hedgehogs is worrying and constitutes a public health risk, given frequent exposure of humans to hedgehogs and because $C$. ulcerans is the predominant cause of human diphtheria in many European countries (1).

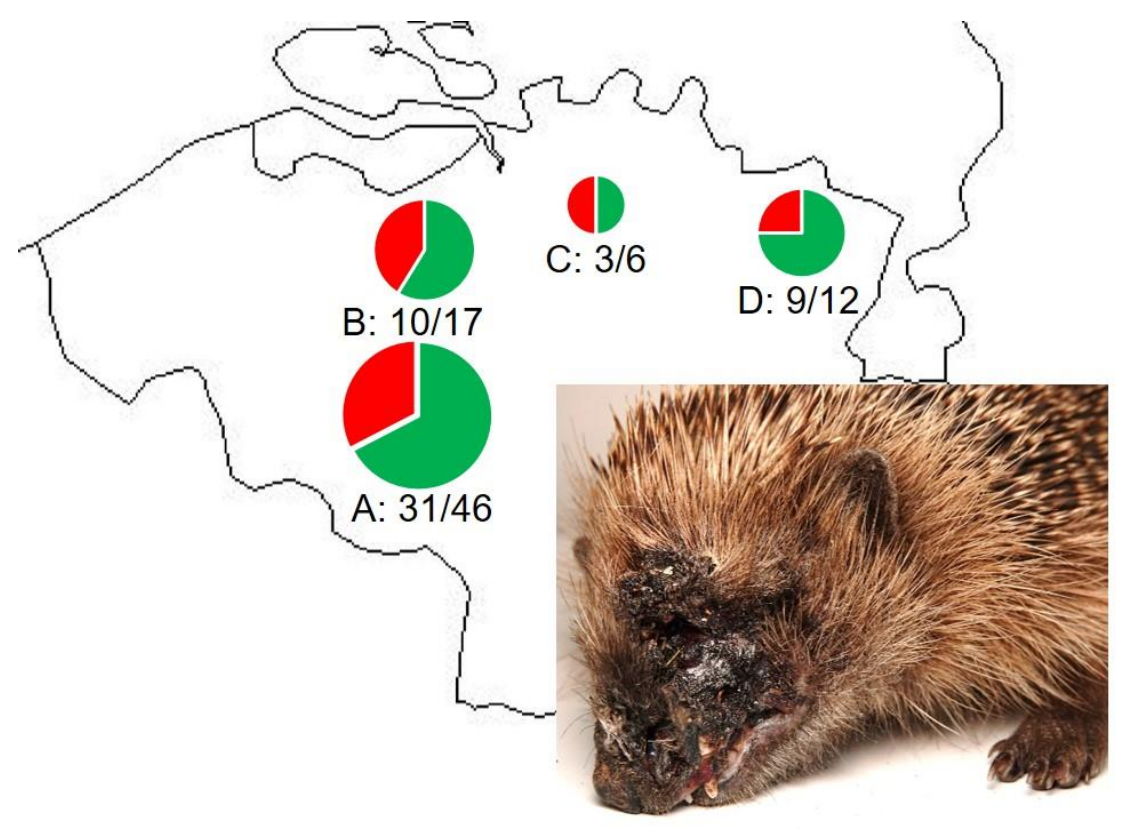

Figure 1. Eighty-one hedgehogs with lesions on the head or limbs from four regions in Flanders were investigated for the presence of $C$. ulcerans (green: proportion of positive animals; red: proportion of negative animals). Regions A: Geraardsbergen, B: Merelbeke, C: Herenthout, D: Oudsbergen. Small picture indicates a representative clinical state with necrotizing facial dermatitis in one male hedgehog from Merelbeke. 
Table 1: Bacterial isolates obtained from 81 diseased hedgehogs across Flanders, Belgium. Numbers reflect the number of positive animals for the respective bacterium. Bacteria were identified using MALDI-TOF mass spectrometry. Biovar identification for the $C$. diphtheriae isolate was performed using the Api Coryne system (BioMerieux).

\begin{tabular}{|l|l|}
\hline Bacterial species obtained from lesions & Number of positive animals \\
\hline Corynebacterium ulcerans & 53 \\
\hline Staphylococcus aureus & 25 \\
\hline Enterococcus faecalis & 23 \\
\hline Streptococcus canis & 18 \\
\hline Streptococcus dys galactiae & 18 \\
\hline Proteus vulgaris/P. hauseri & 17 \\
\hline Staphylococcus rostri & 15 \\
\hline Proteus mirabilis & 14 \\
\hline Streptococcus pyogenes & 13 \\
\hline Staphylococcus xylosus & 10 \\
\hline Staphylococcus microti & 8 \\
\hline Staphylococcus sciuri & 8 \\
\hline Vagococcus fluvialis & 8 \\
\hline Enterococcus avium & 7 \\
\hline Escherichia coli & 7 \\
\hline Staphylococcus pettenkoferi & 7 \\
\hline Morganella morganii & 6 \\
\hline Staphylococcus fleurettii & 6 \\
\hline Pasteurella multocida & 4 \\
\hline Corynebacterium amycolatum & 3 \\
\hline Bacteroides fragilis & 3 \\
\hline Staphylococcus simulans & 3 \\
\hline Trueperella pyogenes & 3 \\
\hline Vagococcus lutrae & 2 \\
\hline Arcanobacterium haemolyticum & 1 \\
\hline Bacillus sp. & 1 \\
\hline Bacteroides pyogenes & 1 \\
\hline Corynebacterium confusum & 1 \\
\hline Corynebacterium diphtheriae mitis & 1 \\
\hline Enterococcus hirae & 1 \\
\hline Enterobacter hormaechei & 1 \\
\hline Gemella haemolysans & 1 \\
\hline Lactococcus garvieae & 1 \\
\hline Streptococcus gallinaceus & \\
\hline Streptococcus thoraltensis & 1 \\
\hline
\end{tabular}

\section{Corynebacterium ulcerans isolates from hedgehogs belong to several clusters}

Fifty-six isolates of C. ulcerans, identified on species level using MALDI-TOF MS (2) and sequencing of the $r p o B$ gene [3], were typed by analysis of infrared spectra (4). The isolates grouped with $C$. ulcerans strains from humans and other animals (hedgehogs and red foxes 
from Germany) $(5,6)$ and clustered in three sublineages (Figure 2). Results argue against emergence and spread of a single $C$. ulcerans clone in the hedgehog population in Flanders. Instead, the high diversity of the isolates suggests $C$. ulcerans endemism in the hedgehog population.

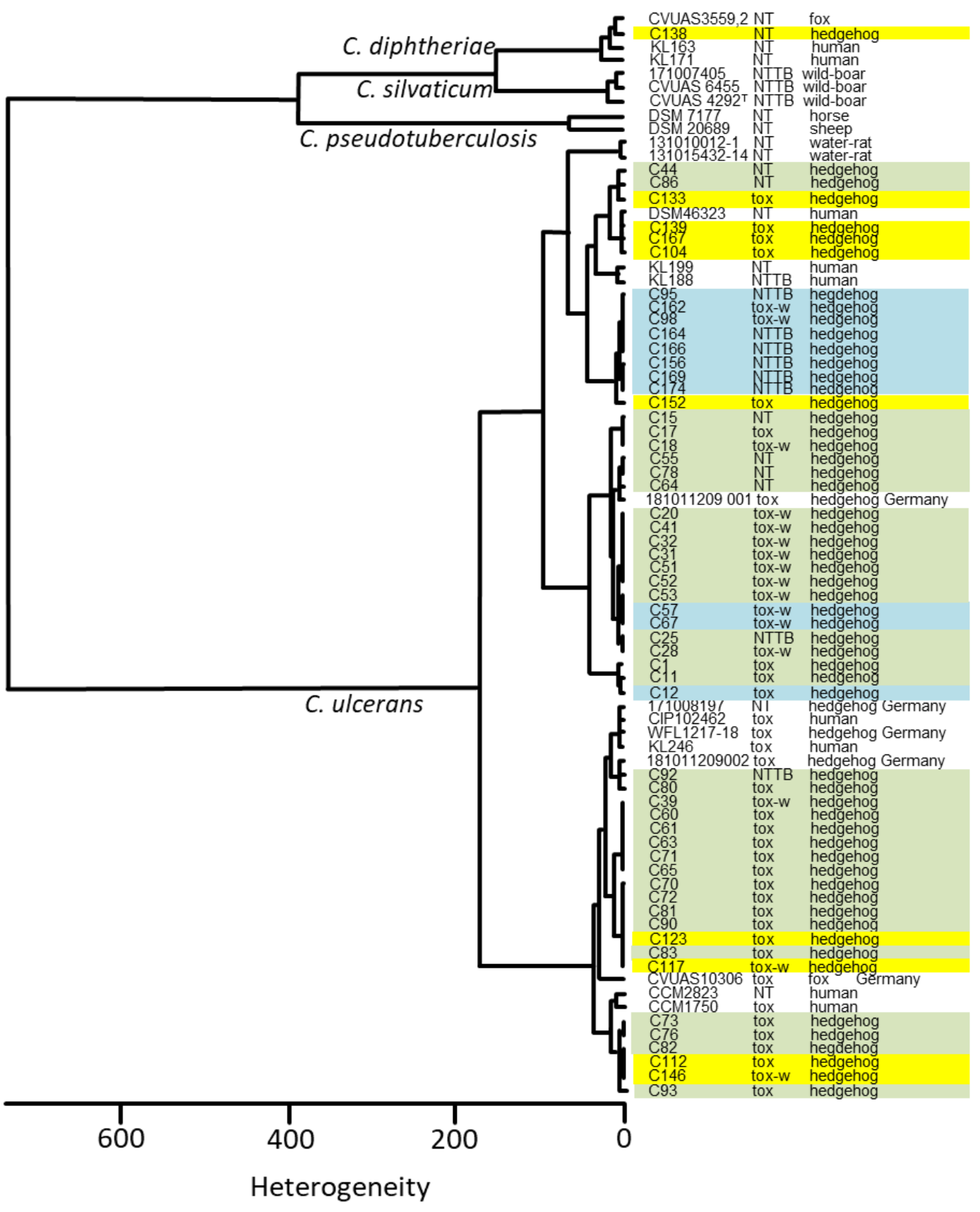

Figure 2. Dendrogram of the FT-IR spectra of Corynebacterium sp. strains obtained from hedgehogs in comparison with spectra from several C. ulcerans isolates, including isolates from other free roaming animals and humans [cf. 8]. Region: Geraardsbergen, green; Merelbeke, blue; Oudsbergen, yellow. tox: toxigenic; tox-w: toxigenic (weak), NTTB: non toxic but tox bearing; NT: no tox-gen. Further details of the isolates are shown on MALDI-UP (7). 


\section{Limited indications of acquired antimicrobial resistance in Corynebacterium ulcerans} isolates from hedgehogs

Minimal inhibitory concentration (MIC) data of all C. ulcerans isolates are presented in Table

2. Acquired resistance against enrofloxacin and spiramycin was noticed in 4 and 1 isolates respectively.

Table 2. Distribution of MIC $(\mu \mathrm{g} / \mathrm{ml})$ values in the 60 isolates of Corynebacterium ulcerans isolates from this study as obtained by epsilometer (ETEST, bioMérieux, Marcy l'Etoile, France) on Mueller-Hinton agar plates with $5 \%$ horse blood and $20 \mathrm{mg} \mathrm{l}^{-1} \beta$-NAD (bioMérieux) according to both European Committee on Antimicrobial Susceptibility Testing (EUCAST) and Clinical and Laboratory Standards Institute (CLSI) guidelines $(8,9)$.

\begin{tabular}{|l|l|l|l|l|l|l|l|l|l|l|l|l|}
\hline & $<0.016$ & 0.016 & 0.032 & 0.064 & 0.12 & 0.25 & 0.5 & 1 & 2 & 4 & 8 & 16 \\
\hline Amoxicillin & 1 & 10 & 6 & 8 & 25 & 10 & & & & & \\
\hline Amoxicillin-clavulanate & 2 & 2 & 2 & 12 & 18 & 22 & 2 & & & & & \\
\hline Clindamycin & & & & & & & & 34 & 26 & & \\
\hline Doxycyclin & & & & & 5 & 27 & 13 & 13 & 2 & & & \\
\hline Enrofloxacin & & 4 & 33 & 19 & & & & 4 & & & & \\
\hline Erythromycin & & 1 & 50 & 9 & & & & & & & & \\
\hline Penicillin & 1 & 1 & 4 & 6 & 17 & 20 & 11 & & & & & \\
\hline Spiramycin & & & & 43 & 17 & & & & & & & \\
\hline Tetracyclin & & & & & & 11 & 29 & 19 & 1 & & & \\
\hline Tilmicosin & & & & & & & 1 & 29 & 29 & 1 & & \\
\hline Trimethoprim sulfamethoxazole & & & 9 & 43 & 8 & & & & & & \\
\hline
\end{tabular}

\section{Most Corynebacterium ulcerans isolates from hedgehogs produce toxins}

Presence and expression of toxicity were evaluated by detection of the diphtheria toxin gene (toxE) using a duplex PCR (3) and the Elek test (10). Results showed the presence of the toxE gene in 50/56 isolates and a positive or weak positive Elek test in 26/56 and 16/56 isolates, respectively. One animal carried a toxE gen positive (isolated from a head lesion) and toxE gene negative (isolated from a foot lesion) $C$. ulcerans isolate. Since the presence of the toxE gene and the toxin production are associated with pathogenicity in humans, these results suggest a zoonotic risk of most hedgehog derived C. ulcerans isolates.

\section{Public and animal health risk of ulcerative hedgehog disease}

Hedgehogs are abundant mammals in Europe, frequently observed in both nature reserves and urbanized areas. Due to their defensive behavior, sick animals are easily presented to animal rescue centers by the public, as testified by the large number of animals we examined in a short time frame in this study. The very nature of their spiny defense promotes breaching and 
inoculating the human epidermis with bacteria during handling. As demonstrated in Table 1, several potentially zoonotic bacterial species, including C. diphtheriae and Streptococcus pyogenes are associated with ulcerative lesions in diseased hedgehogs. However, it is the widespread occurrence of toxigenic $C$. ulcerans in the majority of the diseased hedgehogs across Flanders that should prompt authorities to alert all stakeholders, including the public and the animal rescue centers, to take precautionary measures when handling hedgehogs. While vaccination against $C$. diphtheriae protects against $C$. ulcerans disease, exposure to $C$. ulcerans of susceptible people may result in severe disease (11). Recommendations should include wearing protective gloves and cleaning and disinfecting hands and fomites after contact with a hedgehog and vaccination of people who are frequently exposed to hedgehogs. Treatment of infections with pyogenic coryneform bacteria in animals is challenging and the four rescue centers involved in this study reported poor treatment success. The results of the antimicrobial susceptibility testing suggest that this is not due to acquired antimicrobial resistance, but probably rather to insufficiently high antimicrobial concentrations reaching the C. ulcerans bacteria inside pus. Debridement of the lesions should, therefore, be included in any treatment. Euthanasia should be considered for severe cases.

Emergence of $C$. ulcerans in hedgehogs is in line with a growing number of reports of $C$. ulcerans infections in wildlife across Europe and warrants attention across the continent (5, 6, 12, 13, 14). Reports dating back from the 1950s and the presence of several distantly related clusters of $C$. ulcerans in this study argue against a recent introduction of this pathogen in European wildlife populations and favors the hypothesis that the observed and previously unreported, high numbers of diseased hedgehogs result from pathogen emergence from an endemic state. The finding that only male hedgehogs were presented with this disease, and lesions are mostly found on body parts not covered with spines, suggests the $C$. ulcerans infections may be opportunistic infections of wounds, arising from male specific behavior during the mating season. Bite wounds are well known to be prone to infection with opportunistic pathogens that are part of the oral microbiota (15). The strain typing indeed suggests that hedgehogs constitute a significant reservoir of highly diverse $C$. ulcerans isolates. Active surveillance should elucidate the magnitude of this reservoir in healthy hedgehogs and other wildlife. Further studies should unravel the mechanisms underpinning the observed emergence of this zoonotic wildlife disease from its endemic state. 


\section{Acknowledgements}

This work was funded by the Agency for Nature and forests of the Flemish government, the MALDI-TOF MS was financed by the Research Foundation Flanders (FWO-Vlaanderen) as a Hercules project (G0H2516N, AUGE/15/05), the BOF research as a ZAP mandate to A. Martel and the Bavarian State Ministry of Health and Care as well as by the German Federal Ministry of Health via the Robert Koch-Institute (09-47, FKZ 1369-359).

We thank the rescue centers VOC Wilde Dieren (Nancy Van Liefferinge and Filip Berlengee), VOC Merelbeke (Nick De Meulemeester), Natuurhulpcentrum (Frederick Thoelen) and VOC Neteland (via Tom Verbeek) for the participation to this study.

\section{References}

1. Wagner KS, White JM, Lucenko I, Mercer D, Crowcroft NS, Neal S, et al. Diphtheria in the postepidemic period, Europe, 2000-2009. Emerg Infect Dis. 2012;18(2):217-25.

2. Rau J, Eisenberg T, Peters M, Berger A, Kutzer P, Lassnig H, et al. Reliable differentiation of a non-toxigenic tox gene bearing Corynebacterium ulcerans variant frequently isolated from game animals using MALDI-TOF MS. Vet Microbiol. 2019;237:108399.

3. Martini H, Soetens O, Litt D, Fry NK, Detemmerman L, Wybo I, et al. Diphtheria in Belgium: 2010-2017. J Med Microbiol. 2019;68:1517-1525.

4. Eisenberg T, Kutzer P, Peters M, Sing A, Contzen M, Rau J. Nontoxigenic tox-bearing Corynebacterium ulcerans infection among game animals, Germany. Emerg Infect Dis. 2014;20:448-52.

5. Berger A, Dangel A, Peters M, Mühldorfer K, Braune S, Eisenberg T, et al. Toxpositive Corynebacterium ulcerans in hedgehogs, Germany. Emerg Microbes Infect. 2019;8:1, 211-217.

6. Sting R, Ketterer-Pintur S, Contzen M, Mauder N, Süss-Dombrowski C. Toxigenic Corynebacterium ulcerans isolated from a free-roaming red fox (Vulpes vulpes). Berl Munch Tierarstl Wochenschr. 2015;128(5-6):204-8.

7. MALDI-User Platform MALDI-UP. https://maldi-up.ua-bw.de. Accessed 06.07.2020

8. The European committee on antimicrobial susceptibility testing. 2016. Breakpoint tables for interpretation of MICs and zone diameters, version 6.0. http://www. eucast. org/ fileadmin/src/ media/PDFs/EUCAST_files/ Breakpoint_tables/ v_6. $0_{-}$ Breakpoint_table. 
9. CLSI. Performance standards for antimicrobial susceptibility testing; approved standard, M100-S26, vol. 30 No. 1. 2011;30.

10. Engler KH, Glushkevich T, Mazurova IK, George RC, Efstratiou A. A modified Elek test for detection of toxigenic corynebacteria in the diagnostic laboratory. J Clin Microb. 1997;35(2):495-498.

11. Gower CM, Scobie A, Fry NK, Litt DJ, Cameron JC, Chand MA, et al. The changing epidemiology of diphtheria in the United Kingdom, 2009 to 2017. Euro Surveill. 2020;25(11):pii=1900462.

12. Foster G, Patterson T, Howie F, Simpson V, Davison N, Efstratiou A, et al. Corynebacterium ulcerans in free-ranging otters. Vet Rec. 2002; 150:524.

13. Hirai-Yuki A, Komiya T, Suzaki Y, Ami Y, Katsukawa C, Takahashi M, Yamamoto A, et al. Isolation and characterization of toxigenic Corynebacterium ulcerans from 2 closed colonies of cynomolgus macaques (Macaca fascicularis) in Japan. Comp Med. $2013 ; 63: 272-8$.

14. Olson ME, Goemans I, Bolingbroke D, Lundberg S. Gangrenous dermatitis caused by Corynebacterium ulcerans in Richardson ground squirrels. J Am Vet Med Assoc. 1988; 193:367-8.

15. Abrahamian FM, Goldstein EJC. Microbiology of Animal Bite Wound Infections. Clin Microbiol Rev. 2011; 24(2): 231-246. 